\title{
Measuring Change Risk for Organisational Decision Making through a Hierarchical Model Process Approach
}

\author{
Charalampos Apostolopoulos ${ }^{1}$, George Halikias ${ }^{1}$, \\ Krikor Maroukian ${ }^{2}$, and Georgios Tsaramirsis ${ }^{3}$ \\ ${ }^{1}$ School of Engineering \& Mathematical Sciences, \\ City University London, Northampton Square, London, EC1V 0HB, UK \\ ${ }^{2}$ School of Natural \& Mathematical Science, \\ Department of Informatics, King's College London, Strand, London,WC2R 2LS, UK \\ ${ }^{3}$ Department of Information Technology, \\ King Abdulaziz University, Jeddah, 21589, Saudi Arabia \\ \{charalampos.apostolopoulos.1,g.halikias\} @city.ac.uk, \\ krikor.maroukian@kcl.ac.uk, gtsaramirsis@kau.edu.sa
}

\begin{abstract}
Project Management has long established the need for risk management techniques to be utilised in the succinct identification and mitigation of associated risks in projects. Such techniques aim at the reconciliation of countervailing project activities to reduce scope creep, increase the probability of on-time and within-budget delivery. Uncontrolled changes, regardless of size and complexity, can develop risks to projects and affect project success or even an organisation's project delivery coherence. Ideally, a change or consequence based upon a decision should have a fairly high level of predictability and thus a low level of a potential risk materializing, which would significantly undo the decision taken. This paper proposes a novel modeling process approach; CRAM (Change Risk Assessment Model), which could significantly contribute to the missing formality of business models especially in the change risk assessment area.
\end{abstract}

Keywords: CRAM, Change Risk Assessment Model, Project Management, Change Management, Risk Management, Decision Analysis.

\section{Introduction}

Nowadays, a mission critical necessity for an organisation seems to be the adaptation to specific customer requirements and concepts such as: strategic business planning, customer satisfaction, market adaptation and subsequently efficient and effective business change management. However, the transitional period of change is not only time consuming but also a risky process. In this context, risk can be regarded as an integral part for both, businesses and management.

Nonetheless, the processes of change management and risk assessment are usually regarded as separate business domains and ones which should be generally implemented during the whole life cycle of a project. Besides the generic need for 
change, implementing change is often perceived as an unsurpassable challenge due to several organisational barriers and behavioural aspects relating to human resources, who express considerable resistance to change which often hinders the overall process success.

Project management can have added strategic value, when the level of effectiveness and the efficiency with which a project is accomplished are interlinked and when project outcomes can provide overall business value. Furthermore, change management, also poses as a strategic and structured approach to transitioning individuals, teams and organisations from a current state to a desired future state.

The implementation of project management also requires changes, e.g. in the processes, tools, and methods used to fulfil organisational goals [1]. In effect, 'change' for project management can be seen as an integrated process which is related to controlling the project's requirements in an effort to change them so as to eventually place activities in order and conform to customers requirements.

This research paper, attempts to facilitate various organisational change risk factors which can influence project success by introducing a novel modelling approach named Change Risk Assessment Model (CRAM). The introduction of CRAM will allow the identification and definition of speculative relationships, between change risk events in the form of hierarchical risk model analysis.

Further to the introduction, this paper is organised as follows: section (2) presents existing literature findings. Section (3) discusses the methodology used to develop the model, section (4) provides an overview of the CRAM's processes. Research conclusions are presented in section (5) and finally, section (6) defines the next steps to future work.

\section{Literature Review}

There exist many different models and views for managing change, such as the three stage model (Unfreezing, Confusion, Refreezing), [2]; planned change phases (Exploration, Planning, Action, Integration), [3]; managing the transitional phases (Ending, Neutral and New Beginning), [4]. Even though, this work is significant, it corresponds mainly to a narrative and multi-stage process description which excludes any change risk-assessment process.

Change management, is often based on informal models with risk inconsistencies and generalisations that may lead to loss of meaning and semantic gaps. However, models depicting a simplification of reality withhold a significant advantage [5] and in most of the cases models can be used to deduce simplified conclusions about the real world [6].

Taking as example the two most established project management frameworks PRINCE2 $^{\circledR}$ (developed by UK Government) and PMBOK ${ }^{\circledR}$ (developed by Project Management Institute); project success takes into account several significant environmental exogenous and endogenous project factors ([7], [8]). It is clear that any changes in the project constraints as seen in Table 1, can influence the success or failure of the end result of a project or its deliverables. However, it is within the 
Table 1. Project constraints comparison

\begin{tabular}{ll}
\hline PRINCE2 $^{\circledR}$ variables & PMBOK $^{\circledR}$ competing constraints \\
\hline Cost & Scope \\
Timescale & Quality \\
Quality & Schedule \\
Scope & Budget \\
Risk & Resources \\
Benefits & Risks \\
\hline
\end{tabular}

scope of this paper to examine different attributes far beyond the constraints which are extensively referenced in $\mathrm{PMBOK}^{\circledR}$ and PRINCE2 ${ }^{\circledR}$, showing that the four major ones: time, cost, scope and quality are just the peak of the iceberg.

If any one factor changes, at least one other factor is likely to be affected; effectively changing the project requirements or objectives may create in turn additional risks. Taking into account that project management and change management are integrated processes, an analogy can be found between the project and change influence factors. Critical success factors (CSFs) are requirements or deliverables that must have a satisfactory completion rate for the successful outcome of the project [9].

In a relative research [10] with sixty subjects it was indicated that $43.33 \%$ of the sample's respondents agreed that among other factors 'responsiveness to change' is a project success criterion. In a more practical approach [11], a change management toolkit was developed, which actually provides a numerical computation of change risk, irrespective of project management frameworks. The more identified risks before the initiation phase of the project, subject to project complexity, the better the expected outcome can be. Table 2, shows some indicative project risk categories:

Table 2. Project Risk Categories

\begin{tabular}{ll}
\hline Project Risk Categories & \\
\hline Technical & Project \\
Quality & Legal \\
Performance & Environmental \\
Change & Scope \\
Organisational & Quality \\
External / Internal & Schedule \\
Business & Process \\
Cultural & Requirements \\
\hline
\end{tabular}

Changes and associated risks can occur during the whole life cycle of a project; however, it is vital to assess the possibility of success materialisation before the decision is made to proceed with the change or not. CRAM has the capacity to define the internal dynamics of change management within project management eliciting 
also risk cause-and-effect relationships. Effectively, stakeholders are allowed to describe a problem as they see it, refine the complexity and structure a hierarchy of attributes.

\section{Methodology}

The methodology in terms of scientific research used so as to develop the nodes and attributes of the prototype model, combined in depth literature review analysis and personal interviews in correlation with group meetings (Delphi technique). Interviews (high level executives from diversified industries) were proven more than assistive in coupling together not only professional experience, but also the personal reflection of the participants.

Taking into account that focused group discussions (Delphi Technique) was engaged as a further verification tool of the interviews results, it was more than obvious, that a group environment is beneficial for the respondent in gaining a deeper understating of the research questions. Professionals were able to discuss further their common opinions or disagreements; contribute more effectively either by listening to new ideas or even discussing in more depth with fellow participants.

The change risk categories that were identified are summarised in table 3.

Table 3. Identified Change Categories

\begin{tabular}{ll}
\hline Change Categories & \\
\hline Individual & Rules / Regulations \\
Organisational & Evolutionary \\
Cost cutting & Revolutionary \\
Process & Strategic \\
Cultural & Transformational \\
Technical & Proactive /Reactive \\
Planned / Unplanned & Technological \\
\hline
\end{tabular}

In turn, the attributes were categorised based on rationalism, testing theory and practice. The key idea of categorisation was to construct the prototype model in a sense that can be used repeatedly in various industries, minimising any bias as possible.

\section{Change Risk Assessment Model Overview}

Even if project managers, change managers or other stakeholders discuss about change and associated risks; still, there is a lot of room for research improvement in this area.

Within the research scope, models are defined as the representation of a view of an interpreter about an entity or concept from the real world [12]. However, it is not uncommon to do business or perform business related activities without the use of models. Often, the modellers themselves have disappeared, and any knowledge that 
was not captured in the specialised models is inaccessible, forgotten, or written off [13]. This leads to the inability to address key business environment factors. Nevertheless, business models which can be combined and configured with project business seem to be an exploited research area [14].

The significance of CRAM is that, it can be considered as a prototype change risk assessment modelling method that can be applied in various project types' or organisations irrespective of size or complexity as seen below. The method can be tailored to specific customer or project needs, taking into account significant environmental change risk factors (add or delete risk attributes on a per case basis).

Because project's scope and objectives differ, in the same sense it is difficult to identify all potential risks.

- Product and Strategy Management

- Software / Technology Solutions

- IT / Telecoms

- Service Provisioning

- Consulting

- Engineering
- Insurance

- Government

- Retail

- Utilities

- Defence

- Banking

In addition, CRAM can be integrated with contemporary project management frameworks like for example PMBOK ${ }^{\circledR}$ and PRINCE2 ${ }^{\circledR}$. The proposed model attempts to enforce and integrate currently prevailing descriptive risk analysis methodologies, by engaging a semi-quantitative and qualitative change management risk modelling approach.

\subsection{CRAM Processes}

CRAM is composed of three interrelated processes which are continually recorded and monitored. CRAM processes accomplish specific risk objectives, which are applied to projects or at a greater extent to business environments with a view to facilitate and control change risks. A schematic interpretation of CRAM processes can be seen in Fig. 1.

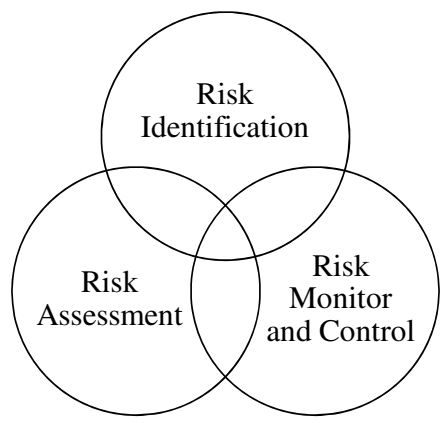

Fig. 1. Change Risk Assessment Model Processes 


\section{Risk Identification}

Risks can be found everywhere and in fact, the difficult part is not to identify but control them. The primary goal of Risk Identification is to recognise the threats and opportunities which may affect the project's objectives and consequently deliverables. Indicatively, risk(s) can be categorised as follows:

Known Risk: this kind of risk refers to an in-depth project analysis which has a considerably high probability of occurrence.

Predictable Risks: are those risks that past experience dictates one may face with high probability.

Unpredictable Risk: is the risk that could happen, but the probability of occurrence in terms, for example, of timing cannot be estimated accurately. The success of many projects is related to the level that this risk will be estimated [15].

Irrespective of risk categorisation, CRAM proposes the following tools and techniques so as to identify change risks:

- SWOT analysis

- Change/Risk surveys

- Delphi technique

- RACI diagrams

- PERT diagrams

- PESTEL analysis

- Risk Breakdown Structure (RBS)

- Interviews

- Brainstorming sessions

Of course, potential risks and required changes can be identified and decided during the entire lifecycle of the project. Nevertheless, they have to be assessed and monitored the sooner the possible.

\section{Risk Assessment}

The basic aim of the proposed model among other objectives is Risk Assessment (Estimation and Evaluation). Change, if uncontrolled can be associated with activities of uncertain outcome(s) which would be deemed unwanted deliverables from project stakeholders. However, when change management and risk management are coupled together, risk aftermath can be reduced; risk is estimated at the planning stage of a project and consequently, there is time to develop a risk mitigation plan and take all necessary preventive actions, acting proactively.

A simple definition of risk in terms of probability of occurrence and its related impact is given by the formula ([9], [16], [17]):

$$
\text { Risk }=\text { Probability } \mathrm{x} \text { Impact. }
$$


The majority of quantitative methodologies use extensively probabilities being rather less ambiguous and imprecise; meaning that they are more objective as far as the assessment of the information and data on identified risks is concerned. The narrative approach of risk estimation has an advantage of providing contextual information but on the other hand does not allow the level i.e. magnitude of risk, to be measured. Qualitative and quantitative scales do indicate levels or rating but lack the information content.

Estimation can facilitate project risks in terms of the probability of occurrence and impact. On the other hand, Evaluation assesses the overall effect of all identified risks aggregated together. Some kinds of risks, like for example financial risks, can be evaluated in numerical terms.

Risk Assessment can be accomplished with the aid of a variety of methods and techniques, such as for example: Simulations, Monte Carlo analysis, CPM (Critical Path Method), AHP (Analytic Hierarchy Process), risk maps, Bayesian probability and statistics, probability trees or even fault tree analysis.

Concerning evaluation, this can be indicatively accomplished for example by a means of benchmark questions, like for example:

- Were all implemented non-standard changes assessed?

- Did the approved changes meet the intended goal?

- Concerning result, does it satisfy stakeholders and more specifically conform to customer requirements?

- Were there found any unplanned changes; which are the associated risks?

- Concerning the implementation phase, did it exceed the project's constraints?

- Are the results documented, for example, in the change risk log?

\section{Risk Monitor and Control}

The Risk Monitor and Control process mainly intends to: identify, analyse, control and track new risks. Risk monitor and control can be accomplished with the aid of a variety of methods and techniques, such as for example:

- Risk Reassessment

- Meetings (consultation, status update)

- Variance Analysis

- Trend Analysis

- $\quad$ Risk Auditing

Alongside the aforementioned CRAM processes an 'Experts' Judgment' may be proven overall constructive. An expert might be, for instance, an individual (project manager, change manager) of a group of executives (Project Steering Committee, Change Advisory Board) which can influence and advice on CRAM results. The hierarchy of CRAM per levels is shown in table 4: 
Table 4. CRAM Nodes Hierarchy

\begin{tabular}{lll}
\hline Level 1 (Root Node) & Level 2 (Parent Nodes) & Level 3 (Child Nodes) \\
\hline Change Risk & Leadership & Performance \\
& Communication & Motivation \\
& Culture & Appraisal \\
& Resistance & Rewards \\
& Requirements & Training \\
& Monitoring & \\
& Flexibility & \\
& Project Management Team & \\
\hline
\end{tabular}

An overall, schematic representation of the prototype risk tree is indicated in Fig. 2, which consists of one (1) core (root) node, eight (8) parent nodes, five (5) child nodes and its respective sixty-one (61) attributes.

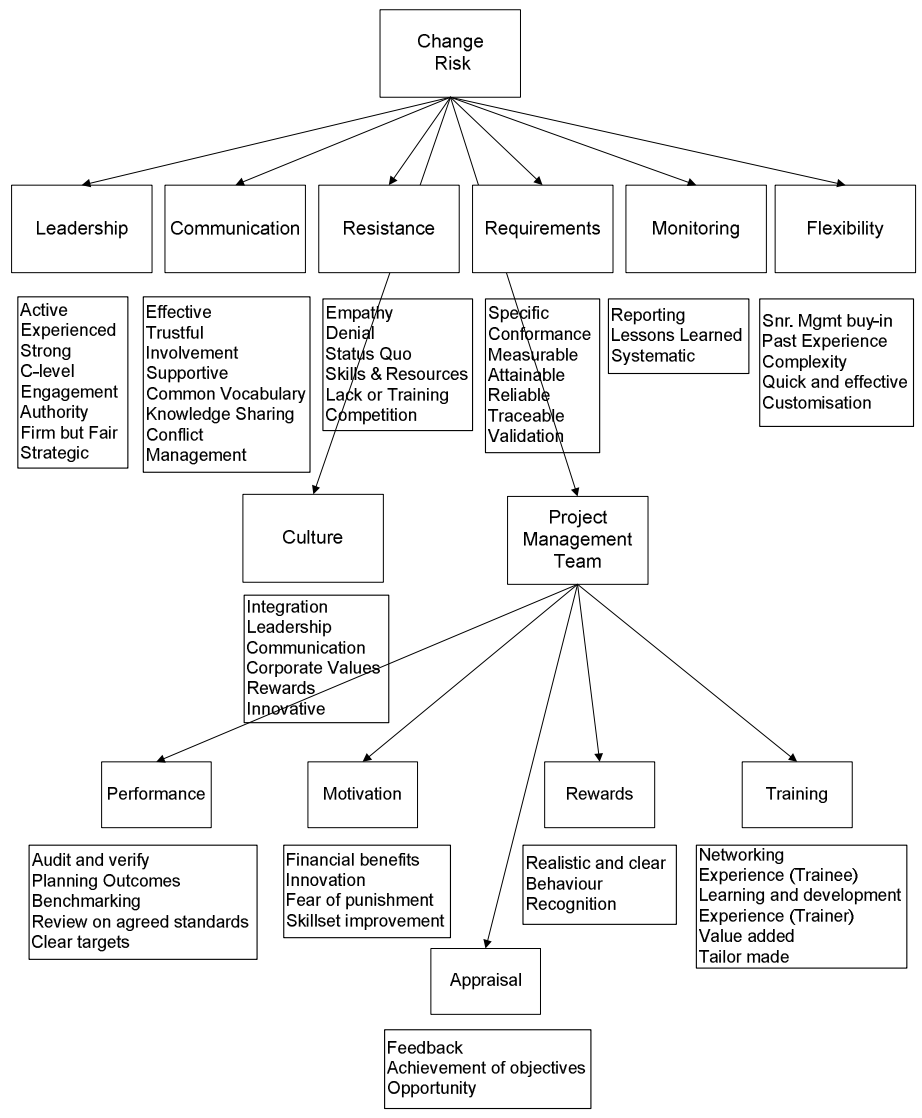

Fig. 2. Change Risk Hierarchy Tree 
Definitions of CRAM's Risk Attributes can be seen at: www.changemodel.net [18].

The 'project management team' is the only parent node possessing child attributes indicating a third level of analysis due to the overall significance in the process of structured project management frameworks. In case a project lacks project management team, then it would be expected to lack coordination and clarity on deliverables.

Depending on the scope and deliverables of a project, CRAM nodes and related risk attribute's hierarchy per level, can change so as to accommodate more of fewer criteria. The only restriction on the hierarchic arrangement of elements is that any element in one level must be capable of being related to some elements in the next higher level, which serves as a criterion for assessing the relative impact of elements in the level below.

\section{Conclusions}

Changes and the process of managing related risks differ among organisations and business cultures. As far as change risk assessment modelling is concerned, there is no one-size-fits-all or all-you-can-eat model. Each customer is different, but what stays the same is the expectation and demand for project success, delivery of services and overall customer's expectations conformance. Each project may require different changes and handling which may be reflected in culture, leadership, decision making, norms and directives and consequently in the general way of implementing and managing projects.

CRAM attempts to take into account various environmental risk factors which influence project success. These risk factors are modeled, and can be assessed numerically in a top-down hierarchical model. Nevertheless, not all risks are the same or have the same priorities. Most of the objectives have to be measured to a certain degree.

On the other hand, there is no unique way to conform to project changes and estimate the relative risks, or to predefine the results of a project. This is because what may seem to be applicable on an individual basis or at a business level, might be inappropriate or insufficient for specific project environments where a number of vendors and clients coexist. Even though, CRAM may carry a degree of complexity on a per case basis, an aim of its scope is to collect data on a global basis, irrespective of specific structured project management framework approaches. In other words, an overall aim of CRAM would be its adoption in project business scenarios on a consistent and repetitive basis so as to identify patterns related to all parent nodes described previously.

Finally, CRAM does not actually dictate any direct or indirect walkthrough; rather it is regarded as a structured approach for facilitating change risk effectively. Even if for example, no project management framework is adopted, CRAM has exactly the same capabilities concerning change risk identification, assessment and monitor and control processes. 


\section{Future Work}

Further to the key integration of Change Management, Project Management and Risk Management in terms of CRAM, future research efforts, will focus on the integration (numerical assessment) with quantitative analysis techniques. In addition, CRAM outcomes are characterised by environmental factors and attributes which can form the basis of an Environment-feature-driven model composition.

A framework capable of transforming such a model layer to subsequent models of lower level abstraction would require a pioneer approach to model-driven initiatives.

Such a framework, resides in the logic behind which the Model Driven Business Engineering (MDBE) framework was developed. In fact, it aims at the unification of model-driven aspects of software engineering and business process modeling, thus, attempting to reconcile the benefits of these two model-driven domains. The outcome could be a more attractive solution to model-driven initiatives for corporate entities whereas academia would have the opportunity to rethink an approach to the study of model based engineering and particularly, model-driven development.

\section{References}

1. Martinuso, M., Hensmen, N., Artoo, K., Kujala, J., Jaafari, A.: Project based management as an organizational innovation: Drivers, changes and benefits of adopting project-based management. Project Management Journal 37(3), 85-97 (2006)

2. Lewin, K.: Field Theory in Social Science. New York Harper and Row (1951)

3. Bullock, R.J., Batten, D.: It's just a phase we're going through: A review and synthesis of OD phase analysis. Group and Organization Studies 10(4), 383-412 (1985)

4. Bridges, W.: Managing Transitions. Addison-Wesley, Reading (1991)

5. Pidd, M.: Five simple principles of modelling. In: Proceedings of Simulation Conference, pp. 721-728 (1996)

6. Williams, T.: Modelling complex project. John Wiley \& Sons (2002)

7. Office of Government Commerce. Managing Successful Projects with PRINCE2 Reference Manual, TSO, The Stationery Office (2009)

8. Project Management Institute. A guide to the project management body of knowledge (PMBOK® guide), 5th edn. (2013)

9. Heldman, K.: Project Manager's Spotlight on Risk Management, Jossey-Bass, A Wiley Imprint, San Francisco (2005)

10. Bryde, D.: Project management concepts, methods and application. International Journal of Operations and Production Management 23(7), 775-793 (2003)

11. Carnall, C.: Managing change in organizations. Prentice Hall, New York (2003)

12. Seidewitz, E.: What models mean. IEEE Softw. 20(5), 26-32 (2003)

13. Denno, P., Steves, M.P., Libes, D., Barkmeyer, E.J.: Model-Driven Integration Using Existing Models. IEEE Software, 59-63 (2003)

14. Wikström, K., Artto, K., Kujala, J., Söderlund, J.: Business models in project business. International Journal of Project Management 28, 832-841 (2010)

15. Rescher, N.: Risk. University Press of America, Lanham (1983)

16. Kendrick, T.: Identifying and Managing Project Risk, 2nd edn. Amacon, NY (2009)

17. Kerzner, H.: Applied project management. Wiley Publications, New York (2000)

18. Change Risk Assessment (CRAM) Glossary (February 2014), http: / /www. changemodel . net (retrieved) 